\title{
Heat transfer from the collective finning of pipes in heat exchangers under asymmetric boundary conditions
}

\author{
Andrey Aksenov ${ }^{1, *}$ \\ ${ }^{1}$ Moscow State University of Civil Engineering, Yaroslavskoe shosse, 26, Moscow, 129337, Russia
}

\begin{abstract}
The solution of the problem of the heat flux from the surface of collective finning at various temperatures of its ends is given. The obtained analytical formulas allow giving a quantitative estimate of the influence of the asymmetry of the boundary conditions on the heat transfer through the finned heat exchange surface. Calculated dependencies can be used in the design of high-efficiency heat exchangers for air conditioning, ventilation, and heating systems. The presented results can be especially useful under real operating conditions of heat exchange surfaces. This, in turn, ensures the safe and reliable operation of the building's engineering systems as a whole.
\end{abstract}

\section{Introduction}

In heat exchangers, where heat transfer occurs between gas and liquid media, finned heat exchange surfaces are commonly used. Finning of heat-exchange surfaces allows significantly increasing the intensity of heat transfer, developing compact high-efficiency heat exchanging devices, which is especially important for air conditioning systems in which all the heat produced must be transferred to the atmosphere according to the conditions of their operation. Relatively low values of coefficients of heat transfer from the cooling surface to air force to maximize the area of the heat exchange surfaces, which leads to an increase in weight, size and consumption of the metal (mostly color) of these heat exchangers.

The finned heat exchange surfaces are widely used in boiler technology, heat supply systems, ventilation units, air conditioning units.

The theory of heat transfer through fins of semi-infinite length or at symmetric boundary conditions (equal temperatures of heaters or coolers at the ends, equal flow temperature, washing the surface of the fins, and equal heat transfer coefficients) has been developed quite fully. There are reliable design formulas for this case that allow carrying out design and verification calculations of heat exchangers operating in the given conditions or close to them [1-15].

In practice, these conditions are not always fulfilled, and sometimes they have a pronounced asymmetric character. For example, with the collective laminose finning of the air heater tubes, due to the uneven distribution of the heat carrier along them, or the series connection of the tubes in the multi-row heat exchanger, the temperature of the fin is uneven.

*Corresponding author: teplovern@mail.ru 
Sometimes the surface of a flat fin is washed by heat carriers having different temperatures at each side. Often the heat transfer coefficients between heat carriers and the surface of the fins are unequal. For example, recently, in order to intensify heat exchange in the furnaces of steam and hot water boilers of small and medium power, the so-called gilled finning of pipes is widely used. The continuous gilled finned tubes are also used as partitions forming the boiler flues. The flue gases surrounding these heat exchange surfaces at each side have different temperatures and different heat transfer coefficients.

Thus, it becomes urgent to study heat transfer through finned heat exchange surfaces under asymmetric boundary conditions. In this article, an attempt to give a theoretical solution to this problem is made.

\section{Methods}

Let us consider the problem of transferring heat through a thin fin of a given constant section $f$ with length $h$ located between two end heaters $(a$ and $b$ ) or coolers with different temperatures $t_{a}$ and $t_{b}$ (Figure 1).

The fin surface is washed by a gas stream with a constant temperature $t_{f}$.

Let $t_{a}>t_{b}$, and $t_{f}<t_{b}$, or $t_{a}<t_{b}$, and $t_{f}>t_{b}$ (Figure 2), i.e. the direction of the heat flow from the fin surface to the medium or from the medium to the fin surface along the entire fin length is unambiguous.

The heat transfer coefficient $\alpha$ will be assumed constant. The nature of temperature variation along the length of the edge $t=\varphi(x)$ for the cases indicated above is shown in Figures 1, 2.

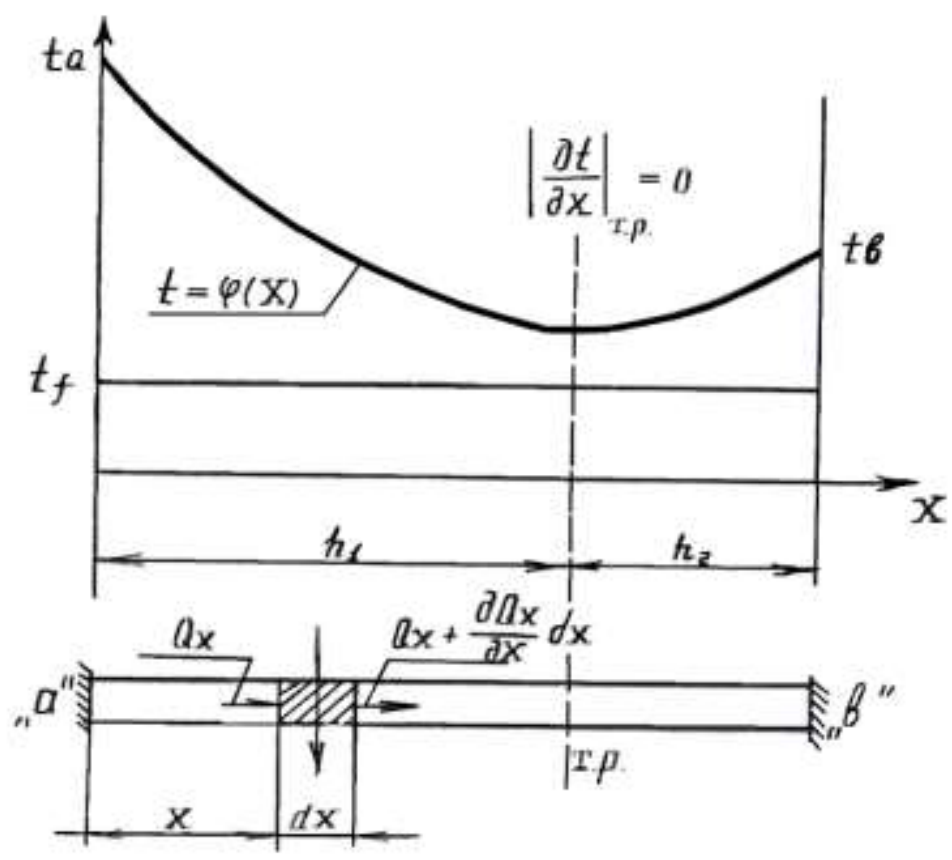

Fig. 1. The temperature distribution along the fin with the end heaters at $t_{a}>t_{b}>t_{f}$. 


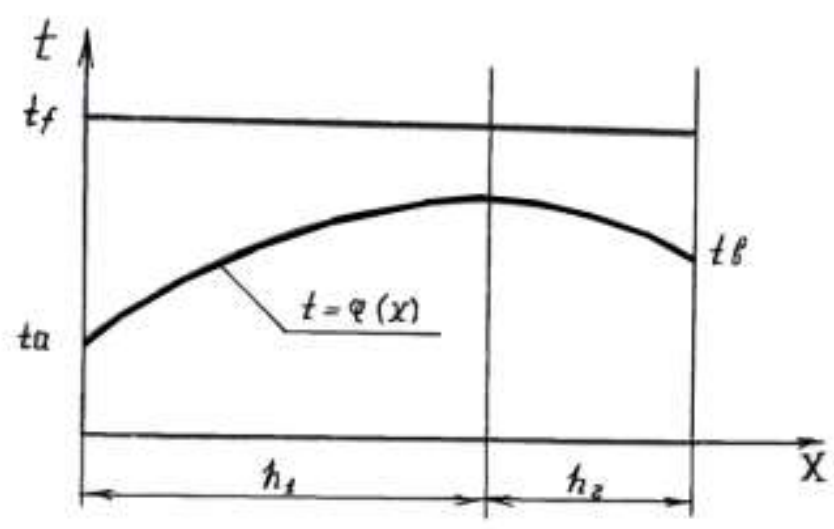

Fig. 2. The temperature distribution along the fin with the end heaters at $t_{a}<t_{b}<t_{f}$.

It is required to establish the analytical law of temperature change $t=\varphi(x)$ and obtain the expression for the total amount of heat $Q$ perceived (given off) by the edge per unit time, i.e. the total heat flux under the conditions specified above.

At an arbitrary distance from the fin base between two cross sections of the fin, we select an element with length $d x$.

\section{Results}

According to the Fourier law, the expression for the second amount of heat entering the selected element through the left cross section by thermal conductivity, we will have the following form:

$$
Q_{x}=-\lambda f \frac{\partial t}{\partial x}
$$

Where $\lambda$ is a thermal conductivity of the fin material, $\mathrm{W} /(\mathrm{m} \cdot \mathrm{K})$.

The amount of heat lost by an element through the right cross section, up to infinitely smalls of a higher order

$$
Q_{x+d x}=Q_{x}+\frac{\partial Q_{x}}{\partial x} d x=-\lambda f \frac{\partial t}{\partial x}-\lambda f \frac{\partial t}{\partial x^{2}} d x .
$$

According to the Newton-Richman law, heat transferred per unit time through the lateral surface of this element as a result of convective heat transfer:

$$
d Q_{u}=\alpha\left(t-t_{f}\right) u d x,
$$

where $\quad \alpha$ is a heat-transfer coefficient, $\mathrm{W} /\left(\mathrm{m}^{2} \cdot \mathrm{K}\right)$;

$\mathrm{u}$ is a perimeter of fin cross-section, $\mathrm{m}$.

On the basis of the energy balance for the selected fin element under steady-state thermal conditions, we can write the following equation

$$
Q_{x}-Q_{x+d x}-d Q_{u}=0
$$

or

$$
\lambda f \frac{\partial^{2} t}{\partial x^{2}} d x-\alpha u\left(t-t_{f}\right) d x=0
$$


Introduce the notation $\alpha u / \lambda f=m^{2}$, and rewrite equation (5) in the following form:

$$
\frac{\partial^{2} t}{\partial x^{2}}-m^{2}\left(t-t_{f}\right)=0
$$

The general solution of this linear differential equation will be as follows:

$$
t=c^{\prime} e^{m x}+c^{\prime \prime} e^{-m x}+t_{f}
$$

Coefficients $c^{\prime}$ and $c^{\prime \prime}$ can be obtained from the boundary conditions.

Formulating the boundary conditions, we divide the fin into two parts: left with length $h_{1}$ and right with length $h_{2}\left(h_{1}+h_{2}=h\right)$. In section $x=h_{1}$ (heat cross section $\left.\left(t_{0}\right)\right)$, where the derivative $\frac{\partial t}{\partial x}$ changes its sign.

Consequently, for the left-hand side of the fin we have the following boundary conditions:

$$
\begin{array}{cl}
\text { At } \quad x=0 & t=\mathrm{t}_{\mathrm{a}} . \\
\text { At } \quad x=h_{1} & \frac{\partial t}{\partial x}=0 .
\end{array}
$$

According to the formula (7) we have (for the left side of the fin):

$$
\begin{aligned}
t_{a} & =c_{1}^{\prime}+c_{1}^{\prime \prime}+t_{f} \\
\left|\frac{d t}{d x}\right|_{x=h_{1}} & =m c_{1}^{\prime} e^{m h_{1}}-m c_{1}^{\prime \prime} e^{-m h_{1}}=0 .
\end{aligned}
$$

Solving jointly equations (6) and (9), we obtain:

$$
\begin{aligned}
& \mathrm{c}_{1}^{\prime}=\frac{\left(t_{a}-t_{f}\right) e^{-m h_{1}}}{e^{m h_{1}}+e^{-m h_{1}}} \\
& \mathrm{c}_{1}^{\prime \prime}=\frac{\left(t_{a}-t_{f}\right) e^{m h_{1}}}{e^{m h_{1}}+e^{-m h_{1}}}
\end{aligned}
$$

Substituting the obtained values of the constants $c^{\prime}$ and $c^{\prime \prime}$ in (7), we obtain

$$
t_{1}=\left(t_{a}-t_{f}\right) \frac{e^{-m\left(h_{1}-x\right)}}{e^{m h_{1}}+e^{-m h_{1}}}+\left(t_{a}-t_{f}\right) \frac{e^{m\left(h_{1}-x\right)}}{e^{m h_{1}}+e^{-m h_{1}}}+t_{f},
$$

or

$$
t_{1}=t_{f}+\left(t_{a}-t_{f}\right) \frac{\operatorname{ch}\left[m\left(h_{1}-x\right)\right]}{\operatorname{ch}\left(m h_{1}\right)} .
$$

At

$$
x=h_{1}
$$

$$
t_{h_{1}}=t_{f}+\frac{t_{a}-t_{f}}{c h\left(m h_{1}\right)}
$$

Heat flow through the left base of the fin:

$$
Q_{1}=-\lambda f\left|\frac{\partial t}{\partial x}\right|_{\mathrm{x}=0}=-\lambda f m\left(c_{1}^{\prime}-c_{1}^{\prime \prime}\right) .
$$

Substituting the obtained values of the constants $c^{\prime}$ and $c^{\prime \prime}$ in (7), we obtain 


$$
Q_{1}=-\lambda f m\left(t_{d}-t_{f}\right) \operatorname{th}\left(m h_{1}\right) .
$$

Formulas analogous to expressions (13) and (16) can also be obtained for the right side of the fin $\left(h_{2}\right)$

$$
\begin{aligned}
& t_{2}=t_{f}+\left(t_{b}-t_{f}\right) \frac{\operatorname{ch}\left[m\left(h_{2}-x\right)\right]}{\operatorname{ch}\left(m h_{2}\right)} ; \\
& Q_{2}=-\lambda f m\left(t_{\mathrm{B}}-t_{f}\right) \operatorname{th}\left(m h_{2}\right),
\end{aligned}
$$

where $0 \leq x \leq h_{2}$ (base " $b$ "-reading), i.e. the variable $x$ in expression (17) varies from $x=0$ at the right base of the fin, where $t_{2}=t_{b}$, to $x=h_{2}$ in heat section, where $t_{2}=t_{h_{2}}$. According to (17)

$$
t_{h_{2}}=t_{f}+\frac{t_{\mathrm{B}}-t_{f}}{\operatorname{ch}\left(m h_{2}\right)} .
$$

As temperatures $t_{h_{1}}$ and $t_{h_{2}}$ belongs to one heat section, then according to (14) and (19)

$$
\frac{t_{a}-t_{f}}{\operatorname{ch}\left(m h_{1}\right)}=\frac{t_{b}-t_{f}}{\operatorname{ch}\left[m\left(h-h_{1}\right)\right]} \text {. }
$$

The obtained equation allows us to find the coordinate of the cross-section of the heat section in the fin, and consequently, the calculated values of the fin lengths $h_{1}$ and $h_{2}=h-$ $h_{1}$.

In case of thermal symmetry, when $t_{a}=t_{b}$, we have $h_{1}=h_{1}=h / 2$.

$$
\begin{aligned}
& \text { Att } t_{b}<t_{a} \text { and } t_{f}<t_{b} \text {, or } \\
& \text { at } t_{b}>t_{a} \text { and } t_{f}>t_{b}
\end{aligned}
$$

the heat section shifts to the base of the fin " $b$ ".

The resultant amount of heat $Q$ is determined by the sum:

$$
Q=Q_{1}+Q_{2} \text {. }
$$

Let us consider the case when the temperature of the medium $t_{f}$ is less than the temperature of one of the fin edges, for example, $t_{a}$, but it is greater than the temperature of the other baset ${ }_{b}$ :

$$
t_{a}>t_{f}>t_{b}
$$

In this case, one part of the fin surface, where $t>t_{f}$, is heat-liberating, and the other, where $t<t_{f}$, is heat-receiving.

As at $x=0 \quad t=t_{a}$, and at $x=h_{1} \quad t=t_{f}$, then according to (7):

$$
\begin{gathered}
t_{a}=c_{1}^{\prime}+c_{1}^{\prime \prime}+t_{f} ; t_{b}=c_{2}^{\prime}+c_{2}^{\prime \prime}+t_{f} ; \\
c_{1}^{\prime} e^{m h_{1}}+c_{1}^{\prime \prime} e^{-m h_{1}}=0 ; \\
c_{2}^{\prime} e^{m h_{2}}+c_{2}^{\prime \prime} e^{-m h_{2}}=0 .
\end{gathered}
$$

We have the same equations for determining arbitrary constants $c_{1}^{\prime} ; c_{1}^{\prime \prime} ; c_{2}^{\prime} ; c_{2}^{\prime \prime}$, as in (8), (9), therefore the same solutions of(13), (16), (17) and (18) for

$$
t_{1}(x) ; t_{2}(x) \text {; }
$$




$$
Q_{1}\left(h_{1}\right) ; Q_{2}\left(h_{2}\right) \text {. }
$$

The resultant amount of heat in this case will be

$$
Q=Q_{2}-Q_{1}
$$

Heat sections determined as

$$
t_{o}=t_{f}
$$

i.e. in the place where the direction of the heat flow in the convective heat exchange between the medium and the surface changes its sign.

$$
\frac{t_{a}-t_{f}}{\operatorname{ch}\left(m h_{1}\right)}=\frac{t_{f}-t_{b}}{\operatorname{ch}\left[m\left(h-h_{1}\right)\right]}
$$

\section{Conclusion}

1. The obtained analytical solutions allow giving a quantitative estimate of the influence of the boundary conditions asymmetry on the heat transfer through the finned heat exchange surface.

2. The calculated formulas (20) and (21) can be used both in the design calculations of various heat exchangers with finned heat exchange surfaces and in verification calculations, for example, for analyzing the variation in finning efficiency when symmetry is violated in the boundary conditions during the operation of heat exchangers.

3 . The resulting calculation formulas can be very useful in choosing the optimal design dimensions and the layout of heat exchangers with collective finned surfaces.

\section{References}

1. Ye.N. Pis'mennyy, Heat exchange and aerodynamics of packages of cross-finned tubes (Kiev, 2004)

2. M.V. Ivanov, N.A. Russu, E.I. Lobdenko, Tyumen State University of Architecture and Civil Engineering (2010)

3. V.B. Kuntysh., A.B. Sukhotsky, E.S Sakovich., V.P. Mulin, Tubular ribbed surfaces with intensified heat exchange and technology of their production for air cooling devices of the power complex (Minsk, 2013)

4. V.B. Kuntysh, A.B. Sukhotsky, Intensification of heat transfer by plastic dismemberment of the rolling aluminum fins of bimetallic pipes in the air flow (Minsk, 2017)

5. M.A. Mikheev, I.M. Mikheeva, Fundamentals of heat transfer (Moscow, 1977)

6. A.V. Ovsyannik, Vestnik GGTU 4, 47-51 (2012)

7. A.V. Ovsyannik, Vestnik GGTU 2, 31-35 (2011)

8. A.F. Lagutin, P.F. Stoyanov, Probleme Energetic Regionale 3(32), 91-106 (2016)

9. P.F. Stoianov, A.S. Lagutin, Prac. Of the Conference Compressors - 2009, Slovak Republic, 398-403 (2009)

10. V.I. Knyazyuk, A.E. Lagutin,P.F. Stoyanov, Kholodilnaya tekhnika and tekhnologiya 1(141), 5-9 (2013) 
11. P.F. Stoyanov, A.E. Lagutin, Kholodilnaya tekhnika and tekhnologiya 2(124), 35-41 (2010)

12. S. Singh, K.Sorensen, T. Condra, Proceedings of the $56^{\text {th }}$ sims, Linkoping, Sweden, 383390 (2015)

13. A.B. Sukhotsky, G.S. Sidorik, Intensification of free convection in a single-row finned beam in air cooling devices, Minsk 2, 68-74 (2017)

14. Ye.N. Pis'mennyy, Production and technology 17,14-18 (2010)

15. K.S. Egorov, N.L. Schegolev, Investigation of the characteristics of high-compact plates of finned heat transfer surfaces with a displaced rib, (Moscow, 2012) 